\title{
A Multimodal Analgesic Pathway Improves Postpartum Pain Management Scores as Reflected by HCAHPS scores
}

Jamie D. Murphy ${ }^{1^{*}}$, Michael C. Grant ${ }^{1}$, Christopher L. Wu ${ }^{1}$ and Linda M. Szymanski ${ }^{2}$

${ }^{1}$ Department of Anesthesiology and Critical Care Medicine, Johns Hopkins University School of Medicine, 1800 Orleans Street, Zayed 8-120, Baltimore, MD 21287, USA

${ }^{2}$ Department of Gynecology and Obstetrics, Division of Maternal-Fetal Medicine, Johns Hopkins University School of Medicine, 600 N. Wolfe Street, Phipps 228; Baltimore, MD 21287, USA

*Corresponding author: Jamie D. Murphy, Department of Anesthesiology and Critical Care Medicine, Johns Hopkins University School of Medicine, 1800 Orleans Street, Zayed 8-120, Baltimore, MD 21287, USA; E-mail: jmurphy@jhmi.edu

Received date: August 14, 2018; Accepted date: August 28, 2018; Published date: August 31, 2018

Copyright: $\odot 2018$ Murphy JD, et al. This is an open-access article distributed under the terms of the Creative Commons Attribution License, which permits unrestricted use, distribution, and reproduction in any medium, provided the original author and source are credited.

\section{Abstract}

Background: Postpartum pain is an important issue in obstetrics. A standardized method of monitoring pain scores is provided by the Hospital Consumer Assessment of Healthcare Providers and Systems (HCAHPS) national survey. In an effort to improve pain management ans subsequently improve HCAHPS scores in our obstetrics unit, we implemented a multimodal analgesic pathway with the intent to maximize analgesia while minimizing opioid use.

Methods: Traditional postpartum analgesia methods were replaced with a multimodal pathway, including patientcontrolled epidural anesthesia for the first 12-24 hours after cesarean delivery plus acetaminophen and ketorolac IV as needed. Upon initiation of oral intake, scheduled acetaminophen and ibuprofen are administered. Transdermal lidocaine patches and opioids are added as needed. The oral medication pathway is initiated following vaginal delivery. Baseline and post-implementation responses to HCAHPS pain management questions 1) How often was your pain well controlled? 2) How often did the hospital staff do everything they could to help you with your pain were compared.

Results: Prior to analgesia protocol implementation, pain management scores were below the desired benchmark of 78\%. Post-implementation, responses of "Always" or "Top-box" for Question 1 significantly increased [73\% versus 65\%; $p=0.05]$. Post-intervention Top-box responses for Question 2 increased from 82 to $89 \%$ ( $p<0.03$ ).

Conclusions: The development of a postpartum multimodal analgesic regimen resulted in significantly improved pain management scores, reflected by HCAHPS scores. No other systematic intervention occurred to explain these positive changes.

Keywords: Pain management; patient satisfaction; HCAHPS survey; Postpartum

\section{Introduction}

Pain management remains an important issue in the obstetric care of women. Acute postpartum pain following vaginal delivery affects nearly all women, with over $1 / 3$ reporting the pain as moderate to severe, requiring analgesia [1-3]. After cesarean delivery, the majority of women (up to 78\%) report moderate to severe pain in the first 24 hours, which classifies their pain as inadequately treated [4]. In a prospective study comparing pain intensity on postoperative day \#1 among surgical specialties, obstetrics patients reported the worst pain intensity and highest pain during movement. When comparing 179 operative procedures, cesarean delivery pain was ranked 9th (with \#1 being the worst) with the mean worst pain intensity (6.14 out of 10 on a numeric rating scale, NRS) during the first 24 hours postoperatively [5]. In addition to decreasing patient satisfaction, delaying ambulation, increasing post-operative complications, and increasing the risk of developing chronic pain, inadequately controlled pain after cesarean delivery may also negatively affect breastfeeding/infant care and has been associated with postpartum depression [6].
From a hospital administrative perspective, pain control has become an important issue as poor postoperative pain control adversely affects Hospital Consumer Assessment of Healthcare Providers and Systems (HCAHPS) scores [7]. HCAHPS scores are standardized, publicly reported survey results that measure patients' perceptions of their hospital experience enabling comparisons across hospitals - locally, regionally, and nationally - on domains that are important to consumers. The Centers for Medicare \& Medicaid Services (CMS) requires hospitals use these surveys. Because the reports are public, the results may incentivize hospitals to improve their quality of care. The survey also has financial implications as scores are linked to reimbursement of the 32 total questions, 21 address patient perspectives on their care covering 9 topic areas including communication with doctors and nurses, responsiveness of hospital staff, pain management, communication about medicines, discharge information, cleanliness and quietness of the hospital, and transition of care [8].

With nearly 4 million births annually in the United States, and approximately $32 \%$ of them via cesarean delivery [9], the problem of inadequate pain control in this population is important and needs to be addressed. The primary purpose of this study was to determine whether the implementation of a multimodal analgesic pathway on our obstetric unit would improve postpartum pain scores, resulting in a 
positive impact on the hospital's HCAHPS scores. Our primary outcome measures were 2 pain management HCAHPS scores.

\section{Methods}

\section{Development of the Multimodal Analgesic Pathway}

In order to better address pain management as well as to reduce inter-provider practice variation, our team sought to develop a single unified postpartum multimodal analgesia pathway. Traditional postpartum analgesia at our institution involved the following approach after cesarean delivery: pain was treated primarily through the use of patient-controlled epidural analgesia (PCEA) utilizing an infusion of $0.0625 \%$ bupivacaine and fentanyl ( 5 micrograms $/ \mathrm{ml}$ ) plus oral analgesics. Oral medications included acetaminophen $650 \mathrm{mg}$ every 4-6 hours on an as needed (PRN) basis, ibuprofen $600 \mathrm{mg}$ every 4-6 hours PRN, and/or oxycodone 5-10 mg every 4-6 hours PRN per individual provider discretion. Following vaginal delivery, pain was treated in a similar fashion aside from the use of epidural analgesia. below:

The updated postpartum multimodal analgesic pathway is outlined

1. Immediately after cesarean delivery, when the patient is typically nil per os (NPO):

PCEA is initiated $(0.0625 \%$ bupivacaine continuous infusion at 4-6 $\mathrm{ml}$ per hour plus demand dose of $4 \mathrm{ml}$ every 10 minutes). PCEA is maintained as the primary analgesic for the initial 12-24 hours.

All patients receive acetaminophen 1 gram intravenous [IV] as well as ketorolac 30mg IV every 6 hours PRN for breakthrough pain, unless contraindicated.

If there is a contraindication to epidural, inability to place an epidural or pain is poorly controlled (repeated score $>5$ on a selfreported scale of 1-10), a hydromorphone IV PCA is initiated.

2. At the time the patient initiates oral intake and two hours prior to the discontinuation of PCEA:

A formal oral multimodal analgesic regimen is initiated with scheduled acetaminophen 1 gram by mouth (per os; PO) every 8 hours and ibuprofen $600 \mathrm{mg}$ PO every 6 hours.

Patients who experienced breakthrough pain despite the above scheduled combination of acetaminophen and ibuprofen are prescribed a transdermal lidocaine patch, 1-2 patches every 24 hours.

- Patients reporting moderate breakthrough pain (4-6 on numeric rating scale [NRS]) are offered oxycodone 5 milligrams PO every 4 hours; those reporting severe pain (7-10 on NRS) are offered oxycodone $10 \mathrm{mg}$ orally every 4 hours. Hydromorphone $2 \mathrm{mg}$ orally every 4 hours is utilized if oxycodone is insufficient.

The above pathway is almost entirely similar following vaginal deliveries aside from the use of epidural analgesia. Of note, we did not authorize the use of any combination products that included acetaminophen (i.e. Percocet, Vicodin) to prevent accidental acetaminophen toxicity as our protocol schedules the maximum recommended dose of 3 grams daily.
This updated multimodal analgesia protocol was developed during the first month of fiscal year (FY) 2016 (June 2015) and was formally implemented on August 1, 2015.

\section{HCAHPS Scoring}

Our hospital participates in reporting of HCAHPS, which are provided through Press Ganey Associates, Inc. Individual surveys are mailed using a standardized protocol to a random sample of hospital inpatients, including obstetric patients, between 48 hours and 6 weeks after discharge. Results are abstracted, compiled en bloc and reported in a cumulative fashion on a quarterly basis over each individual FY. Although HCAHPS incorporates numerous questions across several major categories, including nursing and physician communication, staff responsiveness and hospital environment, our study focused on the category related to pain management. Therefore, two questions were principally evaluated:

Question 1: How often was your pain well controlled?

Question 2: How often did the hospital staff do everything they could to help you with your pain?

Patients respond using a 4-point Likert scale: Always, usually, sometimes, never. To be considered satisfactory, respondents had to choose Always; thus, data are provided as a percentage of Always responses. This response is termed "Top-Box" when results are publicly reported and is considered the most positive response [10]. Baseline responses were assessed for these pain-related questions for the period July 1, 2014 to June 30, 2015 (FY 2015) and represent the preimplementation phase. Given the formal multimodal analgesic pathway was instituted in August of 2015, we assessed the responses of these same two questions from July 1, 2015 to June 30, 2016 (FY 2016).

\section{Statistical Analysis}

Data were processed and analyzed with the software programs Excel, v. 14.0 (Microsoft Inc., Redmond, WA) and Stata v. 14.2 (StataCorp, College Station, TX) statistical package. Comparisons were made between means using paired or unpaired Students t-tests for repeated measures. Categorical variables were assessed by the Chisquare or Fisher's Exact test, where appropriate. $\mathrm{P}<0.05$ was considered statistically significant. Statistical analysis was performed with the assistance of an in-house departmental statistician.

\section{Results}

Responses to Question 1 and Question 2 by quarter (cumulatively) at baseline (FY 2015) and after the pain pathway intervention (FY 2016) are represented graphically in Figure 1.

Baseline HCAHPS Scores, FY 2015. There were 214 surveys returned in FY 2015. Prior to analgesia protocol implementation, 65\% of patients responded Always to Question 1 (was your pain well controlled?), below the desired benchmark of 78\%. For Question 2 (did the staff do everything they could to address your pain?), the benchmark was met with $79 \%$ of patients responding Always. These numbers provided the baseline metrics to serve as comparison for post-implementation of the multimodal analgesia protocol. 
Citation: Murphy JD, Grant MC, Wu CL and Szymanski LM (2018) A Multimodal Analgesic Pathway Improves Postpartum Pain Management Scores as Reflected by HCAHPS scores. J Surg Anesth 2: 119.

Page 3 of 5

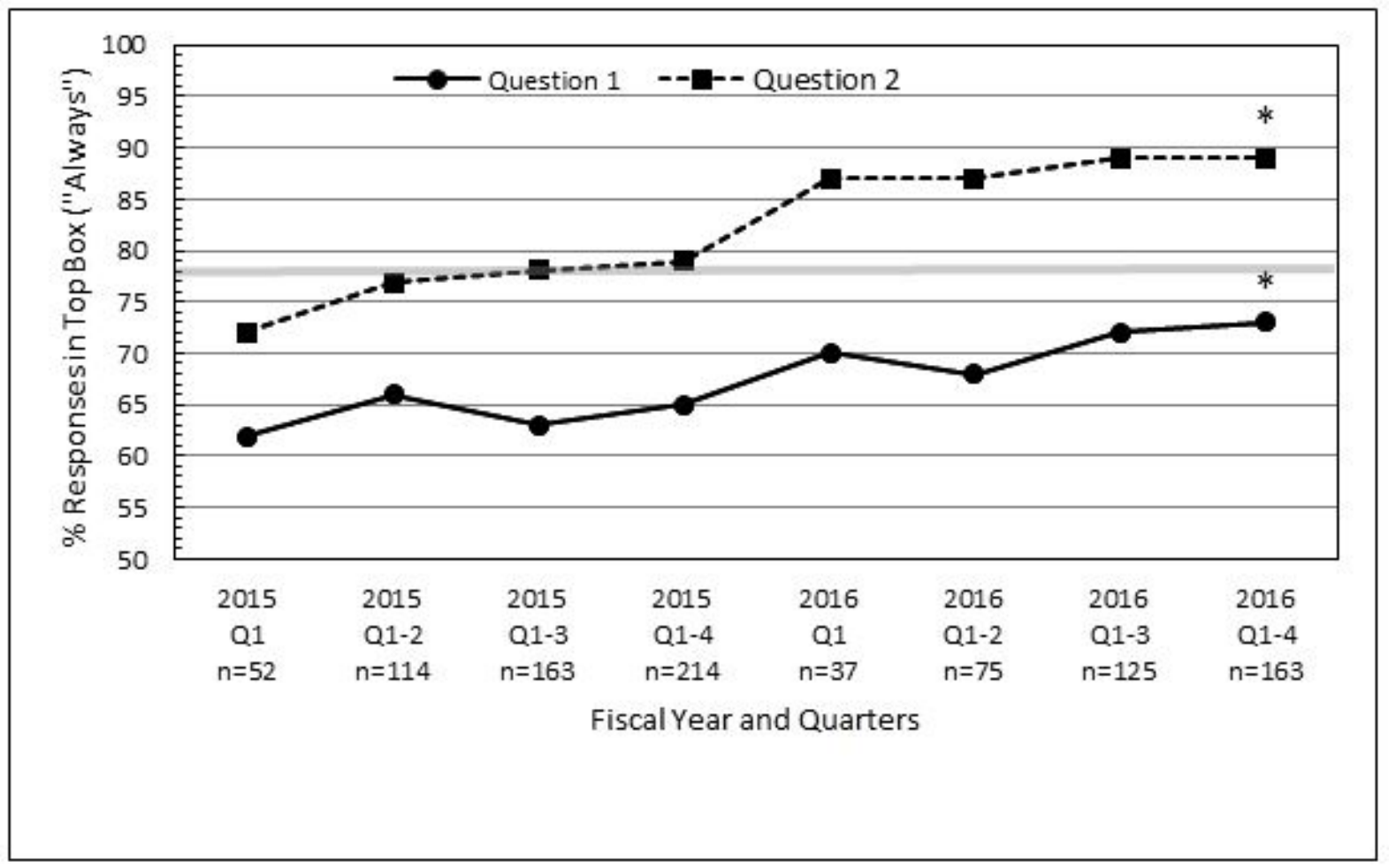

Figure 1: Pain Management Always Response Rates at Baseline and Post-Intervention.

Always (responded with "Always") response rates by quarters at baseline (FY 2015) and post-intervention (FY 2016) for Question 1 and Question 2. Response rates are recorded in a cumulative fashion.

There is an absolute increase in the percentage of Always responses for Question $1(\mathrm{p}<0.05)$. There was also a statistically significant increase in the percent of Always responses for Question 2 for FY 2016 compared to the same question for FY 2015 ( $\mathrm{p}<0.03$ ). In addition, the percent of Always responses for each individual quarter for Question 2 exceeded the desired benchmark (gray line) after the intervention.

*Significantly different from the 2015 Fiscal Year Q1-4 ( $<<0.05)$.

HCAHPS Scores Post-Implementation of Analgesic Protocol, FY 2016. There were 163 surveys returned in FY 2016. The overall percent of Always responses for Question 1 for FY 2016 was significantly increased from the same question in FY 2015 [73\% versus 65\%; $\mathrm{p}=0.05$ ]. While the percent of Always responses to Question 1 did not reach the desired benchmark overall in FY 2016 (73 versus 78\%), improvement was noted over time. WhenQ3/Q4 were analyzed together (rather than cumulatively including Q1/Q2), then $77 \%$ of patients (68 of 88) responded with Always versus 69\% (51 of 75) during Q1/Q2. In addition, when comparing Q3/Q4 in FY 2016 vs Q3/Q4 in FY 2015, there was a statistically significant increase in the percent of Always responses for Question 1 [77 (68 of 88) versus 64\% (64 of 100); $\mathrm{p}<0.01$ ]. There was also a statistically significant increase in the percent of Always responses for Question 2 for FY 2016 compared to the same question for FY 2015 [89\% versus 73\%; p<0.03]. The percent of Always responses for each quarter for Question 2 exceeded the desired benchmark.

\section{Discussion}

The primary purpose of this study was to determine if the implementation of an intervention targeted at improved postpartum pain control would subsequently lead to improvements in HCAHPS scores. Because pain management is included in the HCAHPS survey and our obstetric unit had less than desired HCAHPS scores in FY 2015, we identified pain management as an important area for improvement. Thus, we developed a multimodal analgesic regimen including the combination of scheduled acetaminophen and ibuprofen in addition to transdermal lidocaine patches and opioids on an as needed basis. Pathways were developed for both vaginal and cesarean delivery patients. After initiating this pathway, our pain management scores significantly. Prior to implementing the new multimodal pain management system, our Always or Top-Box HCAHPS scores for Question 1 were in the low 60s and for Question 2 were in the 70s. After implementation of the new pain pathway, our scores were sustained above the desired threshold level determined for the hospital. No other systematic intervention occurred during this time frame to explain these positive changes.

The postpartum analgesic pathway we initiated was adapted from a previously developed multimodal analgesic pathway at our institution for patients undergoing colorectal surgery [11] with a goal of maximizing analgesia and minimizing opioid use. The dose of acetaminophen chosen was higher than we previously administered (1 gm versus $650 \mathrm{mg}$ ) as one randomized trial suggested improved analgesia with a higher dose [12]. This did result in an overall daily dose reduction in acetaminophen from $3900 \mathrm{mg}$ to $3000 \mathrm{mg}$. This lower total intake has been suggested, although not mandated, by the 
Food \& Drug Administration (FDA) [13]. Acetaminophen (both oral and intravenous forms) has been shown to provide postoperative analgesia while minimizing opioid usage and is considered safe for breastfeeding mothers [14]. Nonsteroidal anti-inflammatory agents (NSAIDs) were chosen as they have been shown to provide superior analgesia, even compared to acetaminophen-codeine combinations [15] and are considered relatively safe for breastfeeding mothers [16]. We chose the combination of acetaminophen and NSAIDs as it has been shown to produce superior analgesia compared to either agent alone [17].

Transdermal lidoderm patches were also added to our pathway. Although data on the analgesic efficacy of the patches are equivocal, primarily due to the poor quality of available studies, the risk of adding this medication is relatively minimal. Because the analgesic mechanism of action is different than the other agents prescribed in our pathway, we hypothesized that some patients would experience significant analgesic benefit from this agent [18].

Other analgesics, including gabapentanoids and tramadol, have also been recommended in post-surgical patients. Although there is no large scale high-quality evidence as to the adverse effects of these medications, we felt that the analgesic efficacy of these agents was inferior to that of NSAIDs. Additionally, limited information is available on the safety of many medications with breastfeeding and minimizing medications was an important consideration while developing the multimodal pathway. In response to a Drug Safety Communication by the FDA [19], The American College of Obstetricians and Gynecologists, the Society for Maternal-Fetal Medicine and The Academy of Breastfeeding Medicine recently published a Practice Advisory discussing concerns with the tramadol and codeine postpartum [20], warning that breastfeeding is not recommended while on these medications due to the potential for serious adverse effects in the neonate from opioid overdose. Thus, although these medications have been shown to assist with postoperative pain management, these medications were not routinely administered to our postpartum patients.

Our study has several limitations. This was not a randomized trial. Because we needed to respond to our inadequate pain management, we implemented the new pathway for all patients as soon as it was developed. However, because no other pain management intervention, or other systematic intervention that could directly affect pain management was implemented during this time, we are confident that the multimodal pain management pathway accounts for the majority of the improvement in pain management satisfaction scores. In addition, we do not have demographic data on the patients who returned the questionnaires; however, we do not expect that the demographics of our patient base changed over the study period. Similarly, our data do not distinguish between cesarean and vaginal delivery patients. Again, as this is a random sample of patients who were sent the questionnaire, we do not expect that there were any significant changes in mode of delivery over the two time periods. We are also unable to assess any bias from non-response, as we cannot differentiate among patients who returned surveys from those who did not return surveys or those who did not receive a survey. Further, we had no control over the time frame for when surveys were sent to patients. However, as no changes were made in the manner in which surveys were distributed during the study, no significant changes are expected between the pre- and post-intervention patients. And finally, as our primary purpose was to ultimately evaluate the impact of the new pathway on HCAHPS scores, we do not have detailed information on the type of anesthesia patients received nor do we have other pain scores, such as visual analog scale (VAS) results.

An important factor that may have influenced our pain scores was improved nursing care. With the new pathway, intensive education was provided to the nurses and physicians regarding pain management. Thus, more attention to pain management was likely provided to the patients.

\section{conclusion}

In summary, in an effort to improve patient satisfaction on our obstetric unit, as reflected by HCAHPS scores, with a focus on pain management, we implemented a multimodal analgesic pathway to maximize analgesia while minimizing opioid use. Our pain management scores significantly improved after implementation of the new pathway and have been sustained over time. No other systematic interventions were initiated during this time on the unit. These findings support the use of scheduled acetaminophen and ibuprofen, starting two hours prior to discontinuation of regional anesthesia. Additional pain control modalities include Lidoderm patches and opioid use as needed. This is a relatively simple and inexpensive intervention that appears to enhance patient satisfaction and may also reduce opioid use, which may also have important implications to patient well-being and recovery.

\section{Funding Acknowledgement}

This research received no specific grant from any funding agency in the public, commercial, or not-for-profit sectors.

\section{References}

1. Andrews V, Thakar R, Sultan AH, Jones PW (2008) Evaluation of postpartum perineal pain and dyspareunia--a prospective study. Eur J Obstet Gynecol Reprod Biol 137: 152-156.

2. East CE, Sherburn M, Nagle C, Said J, Forster D (2012) Perineal pain following childbirth: prevalence, effects on postnatal recovery and analgesia usage. Midwifery 28: 93-97.

3. Macarthur AJ, Macarthur C (2004) Incidence, severity, and determinants of perineal pain after vaginal delivery: a prospective cohort study. Am J Obstet Gynecol 191: 1199-1204.

4. Karlstrom A, Engstrom-Olofsson R, Norbergh K-G, Sjoling M, Hildingsson I (2007) Postoperative pain after cesarean birth affects breastfeeding and infant care. J Obstet Gynecol Neonatal Nurs 36: 430-440.

5. Gerbershagen HJ, Aduckathil S, van Wijck AJM, Peelen LM, Kalkman CJ, et al. (2013) Pain intensity on the first day after surgery: a prospective cohort study comparing 179 surgical procedures. Anesthesiology 118: 934-944.

6. Eisenach JC, Pan PH, Smiley R, Lavand'homme P, Landau R, et al. (2008) Severity of acute pain after childbirth, but not type of delivery, predicts persistent pain and postpartum depression. Pain 140: 87-94.

7. Maher DP, Wong W, Woo P, Padilla C, Zhang X, et al. (2015) Perioperative factors associated with HCAHPS responses of 2,758 surgical patients. Pain Med 16: 791-801.

8. HCAHPS: Patients' Perspectives of Care Survey. Centers for Medicare \& Medicaid Services. https://www.cms.gov/Medicare/QualitY-InitiativesPatient-Assessment-Instruments/HospitalQualityInits/ HospitalHCAHPS.html (accessed August 9, 2018).

9. Martin JA, Hamilton BE, Osterman MJK, Driscoll AK, Drake P (2018) Births: Final Data for 2016. National Vital Statistics Reports Vol 67, No 1. 
Citation: Murphy JD, Grant MC, Wu CL and Szymanski LM (2018) A Multimodal Analgesic Pathway Improves Postpartum Pain Management Scores as Reflected by HCAHPS scores. J Surg Anesth 2: 119.

Page 5 of 5

10. Hospital Consumer Assessment of Healthcare Providers and Systems (HCAHPS). http://www.hcahpsonline.org/en/summary-analyses/ (accessed August 9, 2018).

11. Wu CL, Benson AR, Hobson DB, Roda CP, Demski R, et al. (2015) Initiating an Enhanced Recovery Pathway Program: An Anesthesiology Department's Perspective. Jt Comm J Qual Patient Saf 41: 447-456.

12. Qi DS, May LG, Zimmerman B, Peng P, Atillasoy E, et al. (2012) A randomized, double-blind, placebo-controlled study of acetaminophen $1000 \mathrm{mg}$ versus acetaminophen $650 \mathrm{mg}$ for the treatment of postsurgical dental pain. Clin Ther 34: 2247-2258.e3.

13. Krenzelok EP, Royal MA (2012) Confusion: acetaminophen dosing changes based on NO evidence in adults. Drugs R D 12: 45-48.

14. Spigset O, Hägg S (2000) Analgesics and breast-feeding: safety considerations. Paediatr Drugs 2: 223-238.

15. Nauta M, Landsmeer MLA, Koren G (2009) Codeine-acetaminophen versus nonsteroidal anti-inflammatory drugs in the treatment of post abdominal surgery pain: a systematic review of randomized trials. Am J Surg 198: 256-261.
16. Bloor M, Paech M (2013) Nonsteroidal anti-inflammatory drugs during pregnancy and the initiation of lactation. Anesth Analg 116: 1063-1075.

17. Ong CKS, Seymour RA, Lirk P, Merry AF (2010) Combining paracetamol (acetaminophen) with nonsteroidal antiinflammatory drugs: a qualitative systematic review of analgesic efficacy for acute postoperative pain. Anesth Analg 110: 1170-1179.

18. Bai Y, Miller T, Tan M, Law LS-C, Gan TJ (2015) Lidocaine patch for acute pain management: a meta-analysis of prospective controlled trials. Curr Med Res Opin 31: 575-581.

19. Food \& Drug Administration Drug Safety Communication: FDA restricts use of prescription codeine pain and cough medicines and tramadol pain medicines in children; recommends against use in breastfeeding women. https://www.fda.gov/Drugs/ DrugSafety/ucm549679.htm (accessed August 9, 2018).

20. American College of Obstetricians and Gynecologists, Society for Maternal-Fetal Medicine, and The Academy of Breastfeeding Medicine. Practice Advisory on Codeine and Tramadol for Breastfeeding Women. 